$\xi=-1$

\title{
Instruments of Artificial Intelligence in Management of High Technology Production
}

\author{
Mikhail Leizerovich Krichevsky, Svetlana Vladimirovna Dmitrieva, \\ Julia Anatolevna Martynova \\ Saint Petersburg State University of Aerospace Instrumentation (SUAI), \\ 67, Bolshaya Morskaya Str., Saint-Petersburg, 190000, Russia
}

\begin{abstract}
The instruments of artificial intelligence (AI) that can be used in management of high technology production and training students are considered. Specific differences and characteristics of high technology production (HTP) that set certain requirements for such production management are specified. Brief information from the AI methods that include artificial neural networks, fuzzy logic, genetic algorithms and their combinations are given. It is indicated that there is a relation between the level of training masters and the requirements of modern productions. The necessity to use techniques and methods of AI when training students to form their competencies, knowledge and skills that comply with the HTP is explained. The techniques of using AI instruments in the educational process focused on the practical importance of the tasks being solved in such disciplines as HR management, risk management, strategic management, etc. are shown.
\end{abstract}

Keywords: artificial,intelligence, high technology production, educational process, neural networks, fuzzy logic.

\section{Introduction}

High technology production (HTP) is an important part of the economy of any developed country. HTP means production with high absolute and relative (in relation to total production costs) costs for research and development. It is possible to indicate a quantitative criterion for high technology products, for example, [1] notes that high technology products are considered to be such products in whose production the share of research and development costs is at least $3.5-4.5 \%$ in the total costs. This barrier value of the criterion of the high technology of products is not strict and universal: firstly, it differs in various countries; secondly, the methodology for attributing the R\&D costs in different countries is not the same.

According to the analysis of the US Workforce Information Council, the high technology sector of economy can be defined as a branch with a high concentration of employees from the areas of science, technology, engineering and mathematics (STEM) [2]. HTP most often includes such industries as production of electrical and radio electronic equipment, aviation, rocket, space industries, instrument making industry, microbiological industry, informatics industry, etc.

Success of any company, including high-tech ones, depends on various factors and the cost of technologies, goods or services offered by the organization. The success is based on the methods of the company management: how well its leaders control managers and employees of a lower level, and what modern technologies are implemented when managing [3]. HTP is currently characterized by large-scale computerization of works on solving tasks related to planning, technological preparation, automation of design works and production processes. It was facilitated by the massive introduction of modern information technologies and computer complexes to perform the specified works in the man- agement system. The creation of an infrastructure that provides the maximum effect from using advanced management technologies is especially important. Advanced management technologies include instruments and methods of artificial intelligence (AI).

The article is organized as follows. At the beginning, the AI instruments that can be used in the HTP management are considered and analyzed. Then, based on the selected AI models, solutions are given for the most typical tasks that are set when training students. The final part of the article discusses the obtained results and states the areas for the further research.

\section{Methods}

AI is an extremely broad and hardly formalized concept that is associated with representing, obtaining and further manipulating of knowledge. The AI penetrates into various areas of the human activity, supplements and even substitutes their natural intelligence. While many of these areas have been actually invaded over the recent decade, the tasks of industrial HTP were among the first subjected to intellectual methods since it had an easily estimated economic effect [4].

AI technologies have made a significant progress over the recent years. However, companies still perceive AI methods in their initial state. It makes it difficult to assess the true potential impact of the AI on companies and sectors. Nevertheless, the companies implementing the AI in the main part of their business have already seen the potential of the used technologies and expect even greater benefits. The use of AI methods provides companies with considerable competitive advantages as compared to the others that do not pay serious attention to this issue.

When creating the value chain, [5] singles out four areas where the AI actually is dramatically important: 
1. Project: An accurate forecast of demand, optimization of the proposal and the formation of future proposals for the company success.

2. Products: Larger production and simultaneous minimization of time for its maintenance and repair.

3. Promotion: Assigning a real price and deliver of the right message to the desired goal.

4. Loyalty: Providing clients with extensive and personalized benefits.

Most investments in the AI are domestic costs (R\&D expenditures) born by large companies. Large corporate investments are focused on various components of the AI that depend on the companies' interests. For example, such companies as Apple and Google work at developing technologies within the country, although they vary in breadth and purpose of investments in the AI. Amazon works at robotics and speech recognition. BMW, Tesla and Toyota are among those making considerable investments in robotics and machine learning (ML). Toyota has allocated one billion dollars for the creation of a new research institute on using the AI for robotics and self-driving cars. Industrial giants, such as Bosch, GE and Siemens, invest in ML and robotics, and strive to develop specific technologies related to their core activities. In addition, large companies invest great efforts and finances to attract highly qualified specialists. The pool of true experts in this area is small, and Alibaba, Amazon, Facebook, Google and other technical giants have hired many of them.

ML accounts for almost 60 percent of investments. It is AI subset because this is a tool for many other technologies and applications [5]. ML is interpreted as a class of AI methods that is characterized by not a direct solution of the task but training when solving it [6]. The main problem related to the use of ML methods is to develop a solution for assessing whether an observed object belongs to a particular class.

The selection of the right ML algorithm depends on several factors, in particular, the volume, quality and diversity of the data, as well as the answers analysts want to get from these data. Consequently, the selection of the right algorithm is a combination of the analyst's needs, the availability of software and time. Even the most experienced researchers who are specialists in data processing cannot say which algorithm will work best in a particular case.

When interpreting the AI, two paradigms are considered [7]:

- Strong AI - a hypothesis in the philosophy of the AI; according to it, some AI forms can really substantiate and solve problems,

- Weak AI that rejects this possibility.

The strong AI theory assumes that computers can acquire the ability to think and realize themselves, although their thinking will not necessarily be similar to the human. The creation of strong AI systems will take several decades because here the main focus is made on increasing the power of computer systems that in terms of complexity are comparable to the human brain.

Further on, the work considers instruments of the weak AI.

Systems of the weak AI are already actively used in various areas. Functions of the weak AI can be added to the existing systems and give them various "reasonable" features [8]. Practical achievements when using the weak AI are widely known in technology, for example, in robotics, process management, and in transportation; for example, the "smart home" concept where energy, water, heat consumption are regulated by an intelligent system. To a lesser degree, methods of the weak AI penetrate into the management. Such instruments of weak AI as neural networks, fuzzy logic, and genetic algorithms are usually singled out [9]. That is why these technologies will be briefly characterized.

Artificial neural networks (NN) are used to solve problems that cannot be precisely formulated. Much in the NN theory was taken from neurobiology but modeling of the brain functions is an entirely different scientific field. However, NN borrowed some biological analogies from this area. NN can be defined as follows: $\mathrm{NN}$ are parallel distributed structures of processing the infor- mation consisting of separate elements (neurons) that are interconnected by links.

Today the traditional methods of organizing the computing process in a computer are ineffective when solving a number of tasks related to classes that are not formalized or random and require processing of large scale arrays of information, testing a variety of alternative hypotheses, and searches in databases. The use of NN allows involving in computer technology the information processing method characterized for highly organized biological systems, in particular, the principle of parallelism. Unlike traditional computers of the von Neumann architecture, in $\mathrm{NN}$ the role of programming is performed by learning that is interpreted as the change in the state of the neurons themselves and the links between them.

The basic idea of fuzzy logic (FL) is that the intellectual way of reasoning based on the natural language of human communication cannot be described within traditional mathematical formulas. The formal approach is characterized by strict unambiguous interpretation, and everything associated using a natural language has multivalue interpretation. Professor L. Zade, the founder of the modern FL concept, constructed a new mathematical discipline based not on the classical set theory but on the theory of fuzzy sets. Consistently pursuing the idea of fuzziness, it is possible to describe fuzzy analogs of all basic mathematical concepts and to create an apparatus for modeling human reasoning and ways of solving tasks. The apparatus used in this discipline is also rigorous and accurate like in the classical set theory. However, along with the values "truth" and "false" adopted in the classical set theory, it makes it possible to operate with intermediate values.

Researches in genetic algorithms (GA) are based on the attempts to copy natural mechanisms. All living organisms are in an environment. Every organism has a specific genetic material that contains information about it and allows transmitting its properties to the next generations. In the process of reproduction there is a new organism that inherits some of the properties from its parents. The information about these properties is encoded in genes found in chromosomes. In their turn, the totality of chromosomes makes up genetic material - a genotype.

In the process of transferring properties, genes can change. There is a crossing of various parent chromosomes. Along with crossing, there are often mutations, i.e. replacement of individual genes in the chromosome. There is an organism that contains genes of ancestors but differs from organisms of its parents because it has some individual properties. This organism starts living in its native environment. If it turns out that it is well adapted to life, the body can transfer its genetic material to the offspring. Specimen poorly adapted to the environment will not be able to pass their genes on to the next generation. This idea was proposed to solve optimization tasks.

\section{Results}

The development of HTP requires highly skilled specialists and knowledge of the basic AI instruments, the ability to use the appropriate software, to analyze the obtained results, and to take decisions that cause optimal results. Masters graduating from universities in the area of management must have such competencies to become a part of the organization after graduating from the university with the least costs for retraining. Over the recent few years the university where the authors work has formed a tendency to introduce AI methods into various disciplines on training masters in the management area. Below there are solutions of typical tasks in the areas of management, in particular, risk management, human resources management, strategic management, and search for best values. They are solved by using AI tools. Similar tasks are used when preparing for computer case studies, writing course paper, and master's theses. 


\section{Neural Network Classification of the Bor- rower}

The task on assessing the suitability of a borrower (an individual) by using a neural network in the form of multilayer perceptron will be considered. To solve it, the Statistica 12 software package will be used. The Credit applications data file will be taken from it.
The input variables include avg ing (average income), age (age), loan vol (loan amount), and home owner (type of household). The output variable is status that determines the borrower's class $(1-$ to issue a loan, 2 - to refuse a loan). The number of observations in this example is 60 . The fragment of the data is shown in Table 1.

Table 1. Source Data Fragment.

\begin{tabular}{|c|c|c|c|c|c|}
\hline Ser. No. & HOME OWNER & $A V G_{-} I N C$ & $A G E$ & $L O A N \_V O L$ & STATUS \\
\hline 1 & 1 & 53 & 40 & 15 & 1 \\
\hline 2 & 1 & 52 & 43 & 5 & 1 \\
\hline 3 & 0 & 41 & 35 & 6 & 2 \\
\hline 4 & 1 & 70 & 27 & 6 & 1 \\
\hline 5 & 0 & 28 & 36 & 3 & 2 \\
\hline 6 & 1 & 48 & 30 & 12 & 2 \\
\hline 7 & 1 & 38 & 41 & 13 & 1 \\
\hline 8 & 1 & 42 & 35 & 4 & 1 \\
\hline 9 & 0 & 42 & 27 & 7 & 1 \\
\hline 10 & 1 & 61 & 38 & 18 & 1 \\
\hline 11 & 1 & 56 & 38 & 20 & 1 \\
\hline 12 & 1 & 59 & 41 & 11 & 1 \\
\hline 13 & 1 & 38 & 36 & 4 & 2 \\
\hline 14 & 0 & 33 & 39 & 11 & 2 \\
\hline 15 & 1 & 51 & 40 & 4 & 1 \\
\hline 16 & 0 & 40 & 23 & 10 & 2 \\
\hline 17 & 0 & 43 & 26 & 8 & 2 \\
\hline 18 & 1 & 58 & 41 & 9 & 1 \\
\hline 19 & 1 & 49 & 35 & 12 & 2 \\
\hline 20 & 0 & 39 & 31 & 8 & 2 \\
\hline
\end{tabular}

Under such a set of variables 20 networks were considered. Five $\mathrm{NN}$ that were the best by the minimum of the learning error were left. The architecture of the saved networks was a multilayer perceptron (MLP) with a different number of neurons in the hidden layer. After analyzing the error matrix, it was defined that the most suitable network was a MLP 4-5-2 perceptron, i.e. a network with four input neurons, five neurons in the hidden layer and two output layer neurons. During NN learning, the Broyden-FletcherGoldfarb-Shanno (BFGS) algorithm was used. This is an iterative method of solving optimization tasks. The sum of squares (SOS) was applied as a function of errors. Activation functions in the hidden and output layers were selected in the form of a hyperbolic tangent and a sine respectively. Before using the NN in the working mode of the network, the objects that it had already "seen" during the training were alternately presented: \#\#1,13; they defined the clients of the first and second groups, respectively (Table 1). The network classified them correctly: firstly, the values of the characteristics defining the parameters of borrowers were submitted to the network entrance; then the group number in the column with index 1. Status (Fig.1) was formed on the output of the network. Next, the network started working by the classification of new objects. Lines 3 and 4 in Fig. 1 in columns home owner, avg ing, age, loan vol show the values of object features the network has not "seen" during the training. Nevertheless, the NN classifies them and determines the belonging of each new borrower to the selected category.

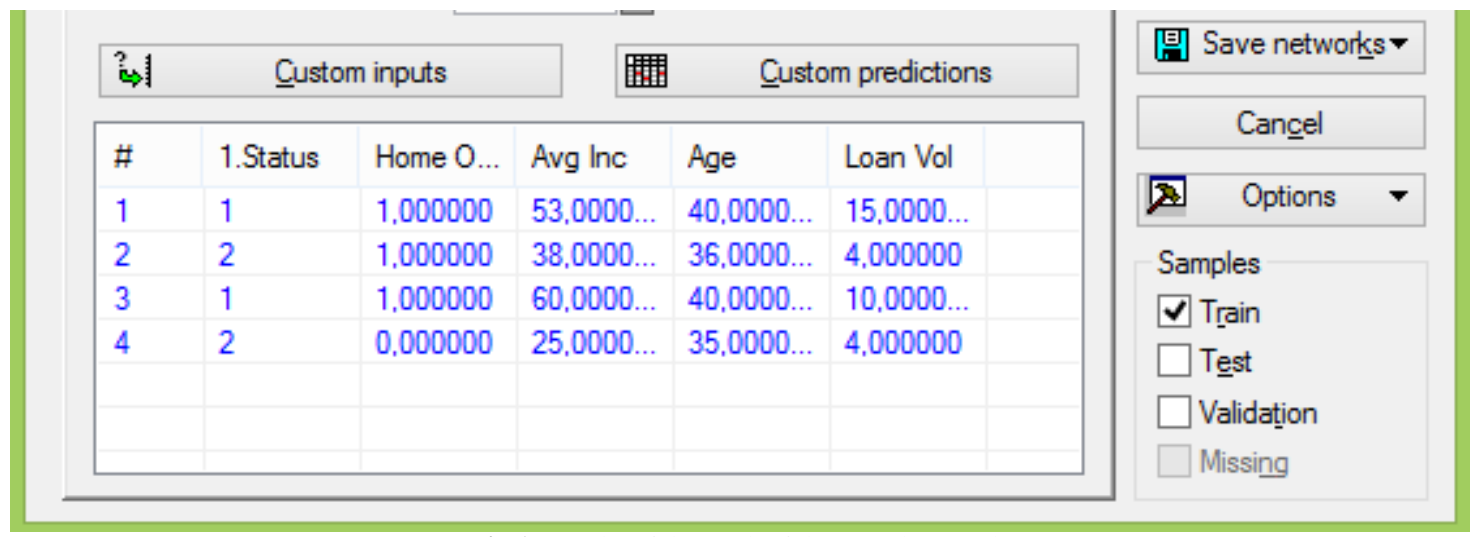

Fig.1. Results of the work of the neural network.

\section{Fuzzy System for Assessing Employee En- gagement}

The HR motivation is the creation of the conditions when the employee gets a need to work to the best of their abilities and strives to achieve heights in their profession. The head of the organization needs to know the level of HR motivation because he can change the strategy of human resource management (HRM) and form the required degree of motivation. Motivation is one of the most important and difficult factors for measuring. It should be taken into account during hiring employees and subsequent formation of an HRM system at an enterprise.

In such situation it is important to have an assessment of HR motivation (HRMA) expressed in the quantitative form because a verbal description of the situation is not enough to make a deci- 
sion about changing the HR strategy. Traditional methods of HRMA do not explicitly provide one value that allows saying about the level of HR motivation.

It is necessary to note that enterprises differ by their form of ownership, industry, number of employees and other characteristics. That is why it is difficult to find the same set of factors suitable for all situations that occur in practice. When solving the task of assessing motivation, it is desirable to reduce the number of influencing factors, otherwise the initial solution will be cumbersome and difficult to interpret. In addition, the diversity of enterprises that differ by many parameters does not allow using the developed method as the only one suitable for HRMA but serves only as a reference point.

To reduce the number of factors, the technique offered in [10] will be used. It indicates four factors that are effective in every firm and influence the HR motivation. These four factors are the following:

- Leadership style,

- Reward system,

- Organizational climate, and

- Structure of work.

Each of these parameters will be characterized in short.

Leadership style. In fact, this factor is the key one in determining the people's attitude to the company and their motivation. Often the change of the leader changes the psychological climate of the company, which causes a change in the work of all employees in the organization.

Reward system. In any organization there is its own kind of a reward system that can differ even in different subdivisions of the same enterprise.

Organizational climate. In the company the organizational climate that characterizes the working atmosphere of the company is created and supported by management. This atmosphere is determined by the employees' attitude to each other, including the relationship of managers with subordinates.
Structure of work. Some types of works require creativity, imagination, and great emotional contribution. However, most works are standardized, routine, uninteresting but necessary for costeffective work of the organization.

The fuzzy inference system for solving this task consists of four input variables specified above, and an output parameter determining the motivation assessment. As it is accepted in the $\mathrm{NN}$, ranges of changes, number of gradations, and types of membership functions (MF) are determined for each of the parameters. In terms of $\mathrm{NN}$ these components form a fuzzification stage.

The work of the created system can be estimated through the "View Rules" option shown in Fig.2. Suppose that the leadership levels, rewards, the degree of the organizational climate, the structure of the work are determined by the values given in the input window at the bottom of Fig.2. Then the degree of motivation of such an employee as determined by the system of fuzzy inference will be approximately 30 points out of 100 . Note that the lower limit of the scale characterizes the lack of motivation, and the upper one - the greatest degree of motivation.

\subsection{Neuro-Fuzzy System for Quantitative Evaluation of the Borrower}

Hybridization of NN with FL allows to significantly increase the efficiency of such neuron-fuzzy systems due to the fact that the disadvantages peculiar to one of the technologies are compensated by the advantages of the other. In particular, NN recognize images well, but the process of the trained network operation is difficult to understand. At the same time, NN systems can explain the conclusions well, but they have limitations on the number of input variables. As a result, it is possible to build hybrid neuron-fuzzy systems where the conclusions are based on NN, and the accessory functions are adjusted with the aid of NN. The advantage of such systems is obvious: the constructed structure is logically transparent.

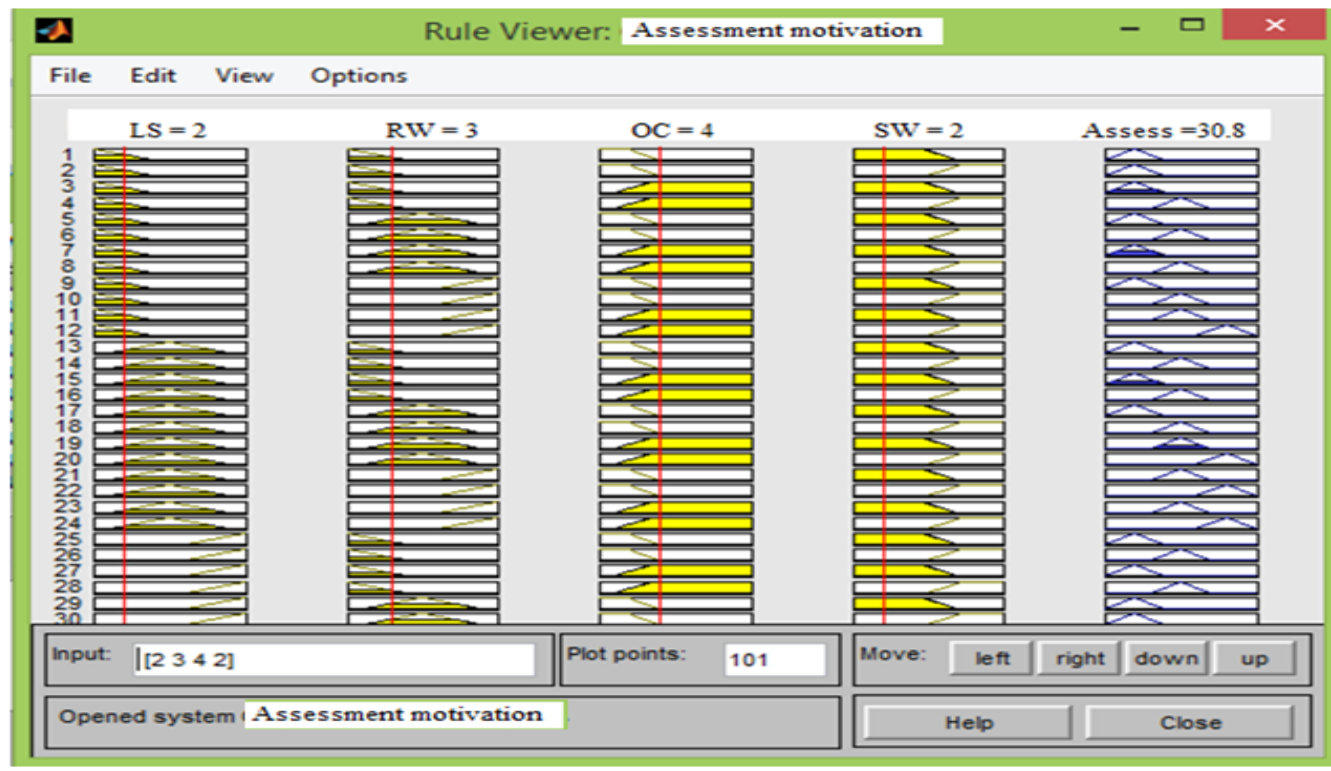

Fig.2 Operation of the Fuzzy Inference System.

The ANFIS (Adaptive Network-Based Fuzzy Inference System) system is one of the first schemes of hybrid neuron-fuzzy networks: a neural network of direct signal distribution of a particular type. The idea of neuron-fuzzy systems is to determine the parameters of fuzzy systems through training methods used in NN. Thus, the ANFIS method has all advantages inherent of fuzzy systems and NN [11].

The FL mechanism can be implemented by using the E. Mamdani or M. Sugeno algorithm. The Mamdani and Sugeno inference systems mainly differ by the way of obtaining a crisp output. In the Mamdani algorithm, a crisp output value is obtained through defuzzification (transition from fuzziness to crispness) of the output fuzzy set. In the Sugeno algorithm, a weighted average value is generated at the output. ANFIS implements the Sugeno fuzzy inference system in the form of a five-layer neural network of direct signal propagation. The purpose of the layers is as follows: - Layer 1 - the terms of the input variables,

- Layer 2 - antecedents (messages) of fuzzy rules,

- Layer 3 - normalization of degrees of the rules implementation,

- Layer 4 - conclusion of the rules, and 
- Layer 5 - the output variable.

Here, the neuron-fuzzy method uses the same data as in 3.1, i.e. with the Credit applications data file from the Statistica 12 package limited to 20 observations.

When using the ANFIS system, it is necessary to have a sample database that can be created by using various methods. Here the following technique will be used: first, the principal components (PC) of the data selection will be calculated, and then, based on the range of the first PC as the initial scale, the status of the borrower (its quality) will be transformed into a more convenient scale from 0 to 10 points. Some loss of information associated with this approach is entirely acceptable because the estimates obtained by the expert way have more errors.
The data selection from 20 observations will be divided into two parts: the training (\#\#1 - 14) part, and the test one (\#\#15-20). After loading all data into the program, a system of fuzzy inference will be created by using the subclustering option. In this method it is assumed that each data point is the potential center of the cluster. The number of clusters is defined during the data distribution algorithm.

Fig.3 shows the ANFIS system for this situation.

As seen, the network has four input nodes, which corresponds to the number of input features. In the first (after input signals) layer the input variables are processed. Each node of this layer defines the linguistic term of the input feature. The next layer is a layer of rules, the inputs of which are the outputs of the first layer.

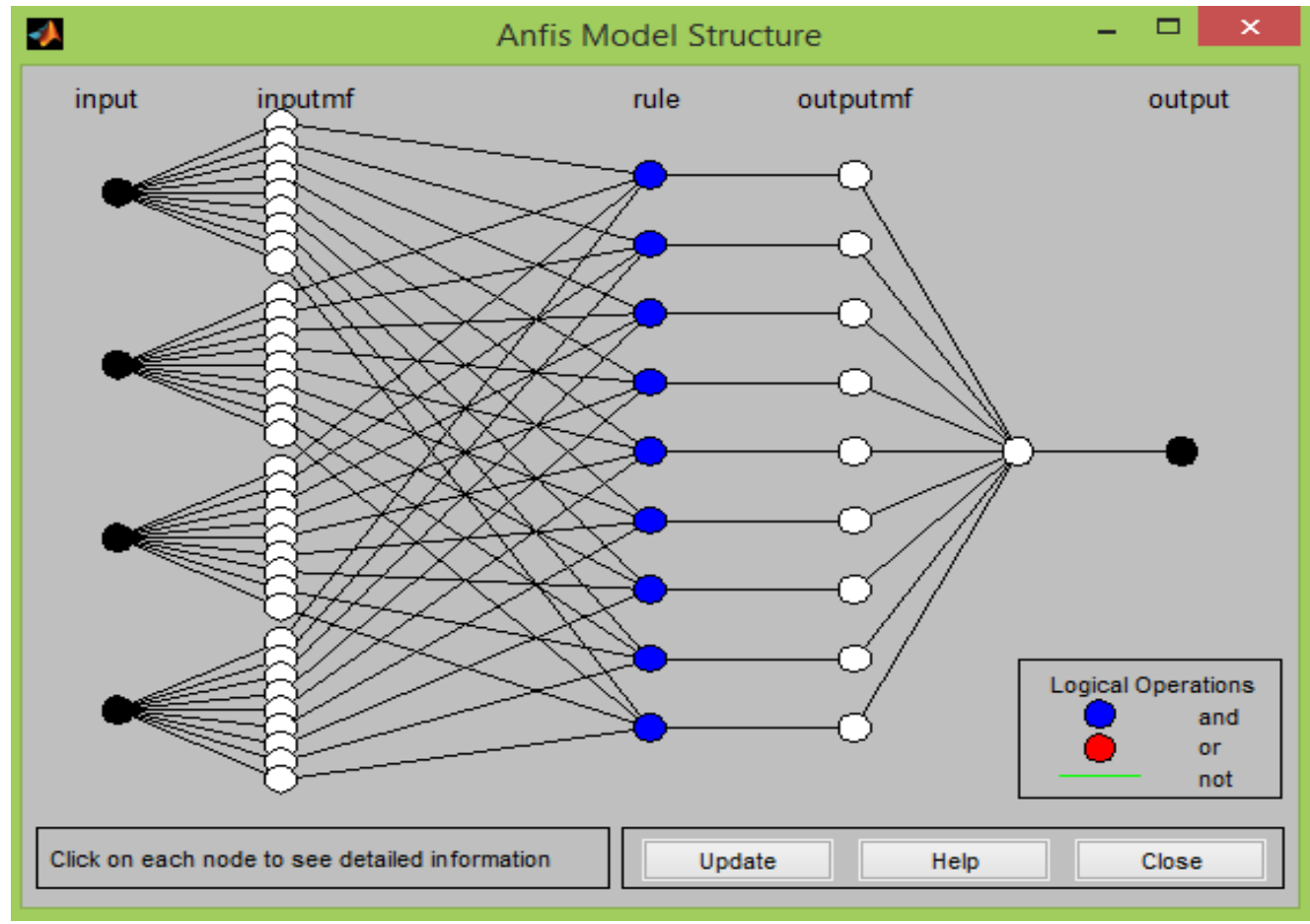

Fig.3. ANFIS System Structure.

As it goes from Fig.3, the system has formed only nine rules. In the third layer, the input values of the neurons of the previous layer are used as input data. Every neuron in this layer calculates the relative degree of fuzzy rule execution. In the pre-last layer, the contribution of every rule to the overall result is determined. Finally, the node of the last layer forms the output value of the ANFIS system.

This system is peculiar by the automatic formation of the rules' base. After that, the training and testing of the ANFIS system by using the Test Now option are carried out. In the situation under consideration, the training selection data coincide with the results calculated by the system. For example, when entering the observation vector [ 15340 15] into the input window, one gets the result that coincides with the previously defined quality assessment of the borrower.

\section{Neural Network Forecasting of Time Series}

In Fig.4, the curve marked as Initial data is the graph of the change in the Aeroflot share price during 120 days (a segment of the series of observations was chosen so that there was growth and decline, an expressed outbreak, noise).

To make a forecast, a multilayer perceptron consisting of the input, hidden and output layers will be used. On the time series (TS) a system of two windows will be formed: the first - wider, where a part of the observed series is introduced, and the second - narrow, it contains only one point and determines the teacher's signal. The TS values from the first window come to the input to the neural network. The number of neurons in the input layer is equal to the number of TS points found in this window. Furthermore, the signals pass through the neurons of the hidden layer and form the output signal of the network. The latter is compared with the teacher's signal determined by the TS value from the second narrow window. At the output of the network, an error is found. It is propagated back through the network (from the output to the input) and adjusts the values of the weights. This is the essence of the network learning by the method of the error back propagation error. Then the system of two windows shifts one point to the right and slides along the row until the narrow window leaves the range: there is no teacher's signal any more, and the network starts making the TS forecast.

The forecast is made in the Statistica 12 program by using a three-layer perceptron. Based on the learning results, the program saves 5 best networks selected by the size of the learning error. The architecture of networks differs only in the number of neurons in the hidden layer: the input and output layers contain one neuron each. This kind of forecast is called the one-step forecast. The network with the lowest learning error will be selected as the main NN. The graphs of the source series and the TS projection obtained by this network are shown in Fig.4. This perceptron will be used to make a forecast for 10 points ahead. The result of the forecast marked as Forecast is also shown in Fig.4. 
Time series projection for Var1

1 steps used as inputs, 1 steps predicted ahead

All samples

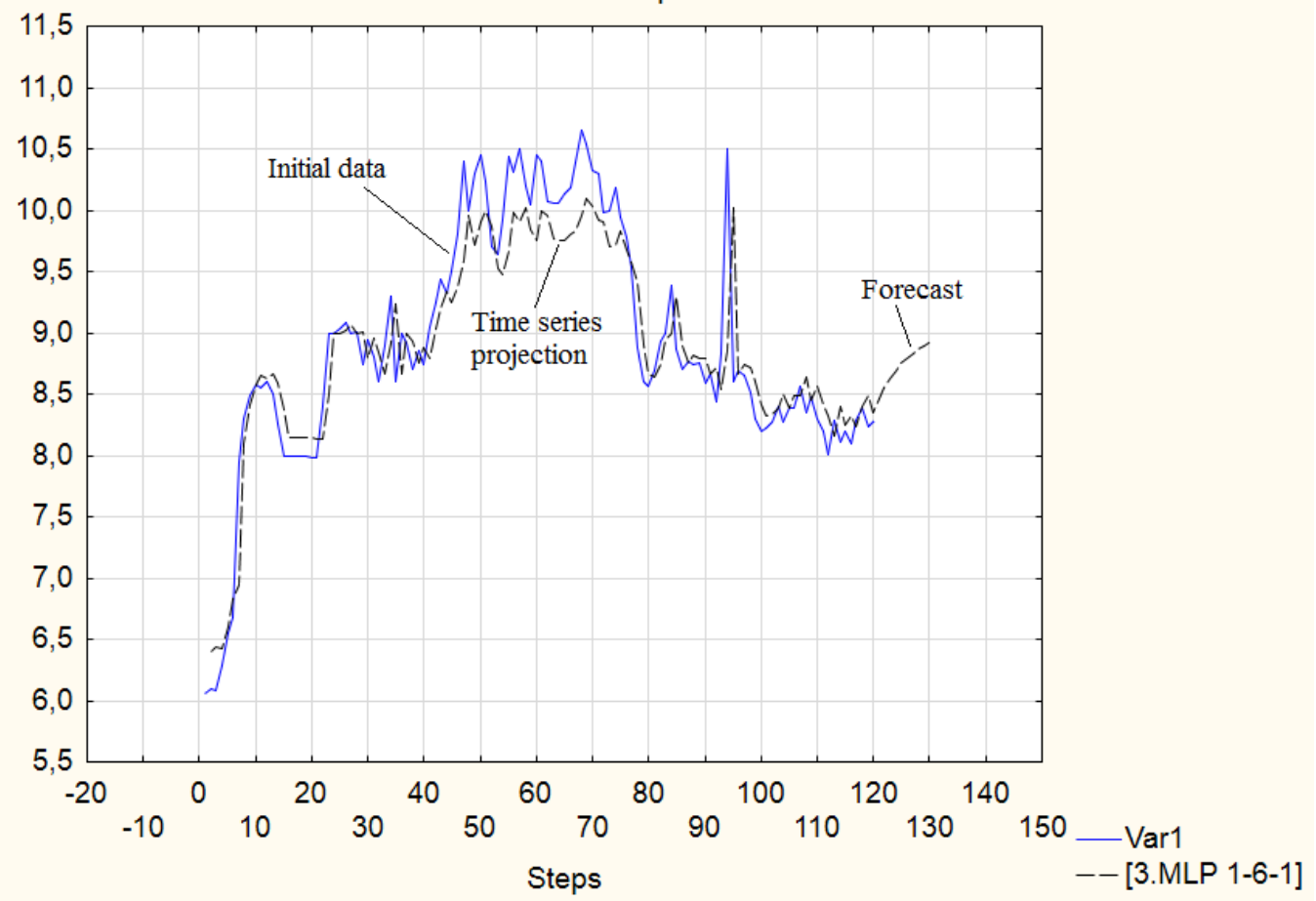

Fig.4. Change of Aeroflot Shares Price.

\section{Genetic Algorithm for Optimum Search}

The genetic algorithm (GA) can be used to solve optimization tasks. The optimization through the GA aims at determining the best possible solution for one or more criteria. To implement the GA, first of all, it is necessary to select a structure to represent these solutions.

Let's assume that the output parameter in a certain management task depends on the two input variables $\mathrm{x} 1$ and $\mathrm{x} 2$, and this dependence is determined by the following expression:

$y\left(x_{1}, x_{2}\right)=x_{1}^{2}-2 x_{1} x_{2}+6 x_{1}+x_{2}^{2}-6 x_{2}$

In the task it is necessary to find such values of the input variables where the output parameter becomes minimal. The Matlab R2012b package will be used to optimize the above target function. In the package there are two possibilities to solve this problem:

- By means of the command window,

- By means of the graphical interface.
The second method will be used. To apply it, the optimtool ('ga') command will open the Optimization Tool optimization window. First, it is necessary to create an m-file in the main Matlab window by using the editor as follows:

function $\mathrm{z}=$ my_fun( $\mathrm{x}$ )

$\mathrm{z}=\mathrm{x}(1)^{\wedge} 2-2 * \mathrm{x}(1) * \mathrm{x}(2)+6 * \mathrm{x}(1)+\mathrm{x}(2)^{\wedge} 2-6^{*} \mathrm{x}$

Save the m-file as my_fun.m.

Then it is necessary to specify the name of the saved file and the number of variables the minimized function depends on in the input window. After that, the Start command starts the calculation process. Fig. 5 shows its results. When the algorithm is terminated, the following messages are found in the Status and results panel:

- "GA terminated";

- The value of the function on the availability of the best specimen in the last generation,

- Reason to stop the algorithm, and

- Coordinates of the last point.

In this task the following results were obtained by using the GA:

The function has reached its minimum, equal to -9.0 , with the values of $\mathrm{x}_{1}=0.01$ and $\mathrm{x}_{2}=3.0$.

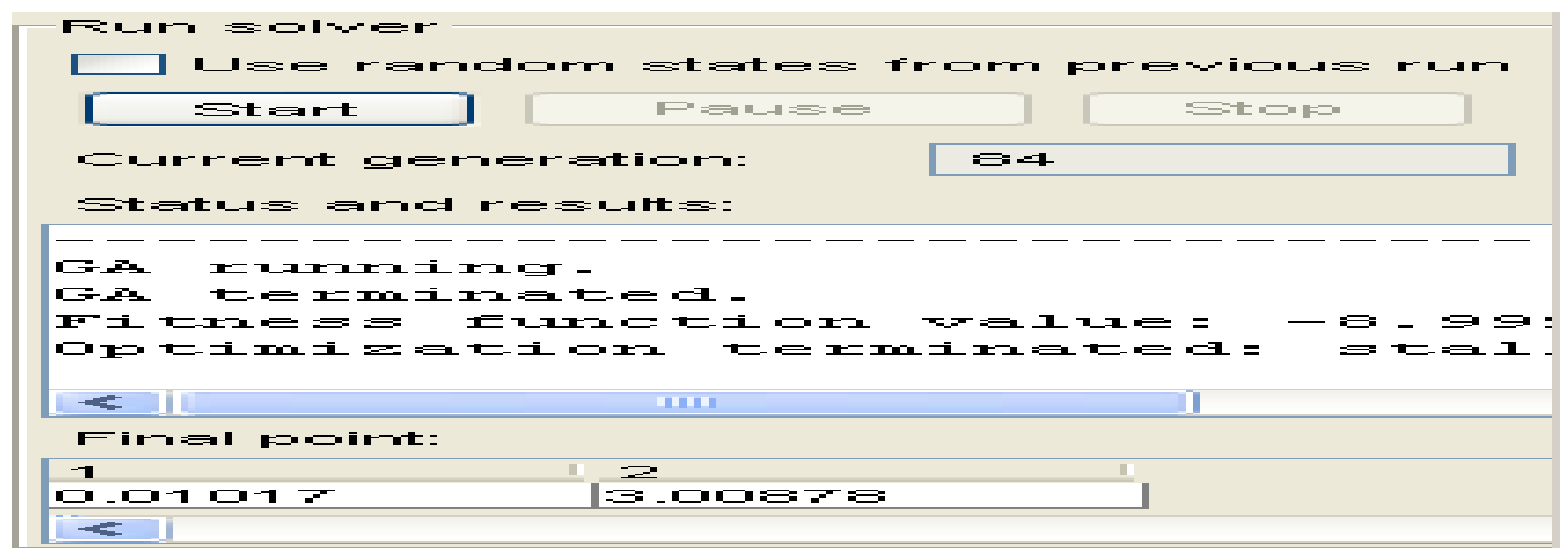

Fig.5. Result of Genetic Algorithm.

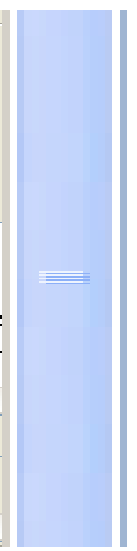




\section{Discussion}

In subsequent works in this area it is necessary to more clearly relate the disciplines for training masters in the field of management and the HTP management. Such relation is obvious because such enterprises must have the specialists whose level of training, competences and skills meet the set requirements. It is necessary to include courses containing the basic methods of $\mathrm{AI}$ in the work programs of the disciplines that are part of the management area.

\section{Conclusion}

The work shows the importance of applying methods of AI in management tasks because this approach allows transforming management as a science from a qualitative description of tasks to a quantitative solution of such problems. The conclusions given in the article show that it is possible to obtain quantitative results in traditional management tasks where earlier the solution results were only qualitative conclusions. The concept of transforming the management disciplines will provide graduate students with the knowledge, competencies and skills required for the further work in the field of HTP aimed at by the world development.

\section{Acknowledgements}

The work was supported by the grant of the Russian Fundamental Research Fund No. 18-010-00338A.

\section{References}

[1]. Priznaki i kriterii otsenki naukoyemkikh rynkov i proizvodstv Features and Criteria for Evaluating High Technology Markets and Productions]. Studbooks. http://studbooks.net/1811888/ekonomika/priznaki_kriterii_otsen ki_naukoemkih_rynkov_proizvodstv. Accessed June 28, 2018.

[2]. M. Wolf, D. Terrell, The high-tech industry, what is it and why it matters to our economic future. Beyond the Numbers: Employment and Unemployment, U.S. Bureau of Labor Statistics 5(8) (2016) 1-7.

[3]. G.P. Peters, Management Strategies for the High Tech Industry. Evaluation Engineering. https://www.evaluationengineering.com/management-strategiesfor-the-high-tech-industry. Revised 2010. Accessed June 28, 2018.

[4]. A. Filchenkov, V. Vyatkin, A. Shalyto, Iskusstvennyy intellekt v proizvodstve vysokotekhnologichnoy produktsii [Artificial Intelligence in the High Technology Production], Springboard to Success 10 (2017) 30-31.

[5]. Artificial intelligence: the next digital frontier? McKinsey Global Institute. https://www.mckinsey.com/mgi/overview/2017-inreview/whats-next-in-digital-and-ai/artificial-intelligence-thenext-digital-frontier. Accessed June 28, 2018.

[6]. K. Wakefield, A guide to machine learning algorithms and their applications. SAS. https://www.sas.com/en_gb/insights/articles/analytics/machinelearning-algorithms.html. Accessed June 28, 2018.

[7]. S. Russell, P. Norvig, Artificial Intelligence: A Modern Approach, Prentice Hall, Boston, 2010.

[8]. M.T. Jones, Programmirovaniye iskusstvennogo intellekta v prilozheniyakh [Artificial Intelligence Applications Programming], DMK Press, Moscow, 2018.

[9]. L. Rutkovskiy, Metody i tekhnologii iskusstvennogo intellekta [Artificial Intelligence Methods and Technologies], Hot Line Telecom, Moscow, 2010.

[10]. B. Tracy, Motivation, AMA, New York, 2013.

[11]. J.-S.-R. Jang, C.-T. Sun, E. Mizutani, Neuro-Fuzzy and Soft Computing: A Computational Approach to Learning and Machine Intelligence, Prentice-Hall, Boston, 1997. 